\title{
Dynamic Incorporation of Wavelet Filter in Fuzzy C-Means for Efficient and Noise-Insensitive MR Image Segmentation
}

\author{
Shang-Ling Jui ${ }^{1}$, Chao Lin ${ }^{1}$, Weichen Xu ${ }^{1}$, Weiyao Lin ${ }^{1}$, Dongmei Wang ${ }^{2}$, Kai Xiao ${ }^{1}$ * \\ ${ }^{1}$ School of Electronic Information and Electrical Engineering, Shanghai Jiao Tong University, \\ 800 Dongchuan Road, Shanghai, 200240, China \\ E-mail: shang_ling_jui@hotmail.com (Shang-Ling Jui) \\ E-mail:forestschao@gmail.com (ChaoLin) \\ E-mail:wcxu@sjtu.edu.cn (Weichen Xu) \\ E-mail:wylin@sjtu.edu.cn (Weiyao Lin) \\ E-mail: showkey@sjtu.edu.cn (Kai Xiao) \\ ${ }^{2}$ School of Mechanical Engineering, Shanghai Jiao Tong University, \\ 800 Dongchuan Road, Shanghai, 200240, China
}

E-mail:dmwang@sjtu.edu.cn

Received 2 December 2014

Accepted 2 May 2015

\begin{abstract}
Image intensity in magnetic resonance (MR) images in the presence of noise obeys Rician distribution. The signal-dependent Rician noise makes accurate image segmentation a challenging task. Although existing fuzzy c-means (FCM) variants with local filters improve the segmentation performance, they are less effective for reducing the negative effect from Rician noise, and the repeatedly applied filter increases their computational intensiveness. To address this issue, we propose a novel image segmentation method which dynamically incorporates wavelet-based noise detector and filter in the FCM membership function. The modified algorithm is designed to exploit both frequency and spatial information in the images and minimizes clustering errors caused by Rician noise. Furthermore, efficiency of the proposed method can be enhanced by the strategy of applying filter only when noise is detected. The experimental results of segmentation on synthetic and brain MR images, demonstrate the computational efficiency and noiseinsensitivity of the proposed method.
\end{abstract}

Keywords: MR images, segmentation, fuzzy c-means, clustering, wavelet, Rician noise.

\section{Introduction}

Magnetic resonance (MR) imaging (MRI) creates much greater contrast between different soft tissues than other imaging modalities such as computed tomography (CT), and becomes the preferred imaging modality for examining neurological conditions that change the shape, volume and distribution of brain tissue. MRI signal further provides multispectral information that reinforce the mapping between brain tissue and the created MR image ${ }^{1,2,3,4}$.

Accurate segmentation of MR images plays a crucial role in many related applications. Since there

\footnotetext{
${ }^{*}$ School of Software, Shanghai Jiao Tong University, 800 Dongchuan Road, Shanghai, China.
} 
is an intuitive similarity between segmentation and clustering ${ }^{2,5,6,7}$, image segmentation methods based on unsupervised clustering techniques are widely used. MR images suffer from the problem of partial volume effect (PVE) in which single image pixel is created by the mixture of signals from several tissues ${ }^{8,9}$; thus it is impractical to label one single group to the affected pixels . Fuzzy clustering becomes more applicable in this scenario, owing to the fact that it assigns one pixel to several groups concurrently based on the membership function 10,11. Among fuzzy clustering methods, the fuzzy c-means (FCM) clustering $12,13,14,15$ is the most widely applied fuzzy clustering technique.

Image pixels in the immediate neighborhood possesses nearly the same feature data. However, this correlation is not fully utilized by the standard FCM algorithm, hence pixels affected by noise could be wrongly grouped. With the dual objectives of utilizing pixel neighboring information and making FCM algorithm insensitive to noise, researchers have proposed various FCM variants which incorporate spatial information into FCM 16,17,18,19,20,21, where mean or median ${ }^{16,17}$, Gaussian ${ }^{18}$ and bilateral filters ${ }^{20}$ are respectively applied. Recent works based on this approach, such as the inclusion of both spatial information and anisotropic neighborhood ${ }^{22}$ and the incorporation of multi-resolution bilateral filter ${ }^{23}$, further enhance the performance.

However the incorporation of spatial filters has two major disadvantages. First, MR image data are usually modeled by the Rician distribution and the noise in MR images obeys a Rician distribution ${ }^{24,25}$. Assuming noise in MR image as Gaussian distribution can lead to an approximately $60 \%$ underestimation of the true noise power ${ }^{25}$. The noise filters applied in the existing FCM variants do not specifically account for Rician noise, and consequently incorporating these filters into FCM algorithm becomes less effective in enhancing the noise-insensitivity. Our previous work addresses this issue by incorporating FCM with a wavelet-based filter for further reducing the negative effect from Rician noise ${ }^{26}$. However, this approach requires the multi-dimensional membership function convolved by the filter in each clustering iteration, thus making the modified algorithm significantly more computational intensive.

In this paper, we present a new method which includes two additional improvements to our previous work ${ }^{26}$. First, in order to enhance the computational efficiency of the algorithm, we propose using the median value of wavelet coefficients as wavelet domain indicator ${ }^{27}$ to detect the existence of Rician noise. When no noise is detected, the algorithm can be accelerated by skipping the unnecessary process of noise removal filtering. Second, we have modified the Rician noise filter to be more suitable for processing Rician noise exhibited in the FCM membership function.. Experimental segmentation results on synthetic images and and multispectral brain MR images, with or without additive Rician noise, suggest that our approach is more efficient and exhibits better clustering performance than comparative FCM variants. Another advantage of the proposed method compared to the most updated FCM variant ${ }^{21}$ is that it does not require any prior knowledge of the images.

The organization of the paper is as follows: in the next section, a brief review of the standard FCM and existing FCM variants is given. We also describe our proposed approach in this section. Experimental data, setting and comparative results obtained within various existing and the proposed FCM variants are presented in Section 3. Concluding remarks and future work proposal are given in Section 4.

\section{Method}

\subsection{Existing FCM Algorithms}

\subsubsection{Standard FCM Algorithm}

With the use of fuzzy memberships ${ }^{12}$, the FCM clustering algorithm minimizes the following objective function:

$$
\left\{\begin{array}{c}
J=\sum_{j=1}^{N} \sum_{i=1}^{c} \mu_{i j}^{m} d_{i j}^{2}=\sum_{j=1}^{N} \sum_{i=1}^{c} \mu_{i j}^{m}|| x_{j}-\alpha_{i} \|^{2}, \\
\sum_{i=1}^{c} \mu_{i j}=1, \forall j=1,2,3, \ldots, N .
\end{array}\right.
$$

where $X=\left(x_{1} ; x_{2} ; \ldots ; x_{N}\right)$ denotes an image with $N$ pixels to be partitioned into $c$ clusters; $x_{i}$ repre- 
sents multidimensional data; $\mu_{i j} \in[0,1]$ represents the membership degree of pixel $x_{j}$ in the $i$ th cluster; $\alpha_{i}$ is the centroid of cluster $i ; m>1$ is the fuzzy index which controls the fuzziness of the resulting partition; $d_{i j}^{2}=\left\|x_{j}-\alpha_{i}\right\|^{2}$ is the distance between pixel $x_{j}$ to centroid $a_{i}$. The cluster centroids $\alpha$ and membership function are shown respectively in (2):

$$
\alpha_{i}=\frac{\sum_{j=1}^{N} \mu_{i j}^{m} x_{j}}{\sum_{j=1}^{N} \mu_{i j}^{m}} ; \quad \mu_{i j}=\frac{1}{\sum_{k=1}^{c}\left(\frac{\left\|x_{j}-\alpha_{i}\right\|}{\left\|x_{j}-\alpha_{k}\right\|}\right)^{\frac{2}{m-1}}}
$$

\subsubsection{FCM with Spatial Filters}

Neighboring pixels in typical images are highly correlated. This spatial relationship is important in clustering, however it is not utilized in the standard FCM algorithm. To exploit the spatial information, a spatial filter is incorporated into FCM algorithm, which can be defined as follows ${ }^{16}$ :

$$
h_{i j}=\sum_{k \in N B\left(x_{j}\right)} \mu_{i k}
$$

where $N B\left(x_{j}\right)$ represents a square window centered on pixel $x_{j}$ in the spatial domain. $\mu_{i k}$ represents the standard membership function of point $x_{k}$ to cluster $i$.

The filter function is included into the membership function in the FCM algorithm as follows ${ }^{20}$ :

$$
\mu_{i j}^{\prime}=\frac{\mu_{i j}^{p} h_{i j}^{q}}{\sum_{k=1}^{c} \mu_{k j}^{p} h_{k j}^{q}}
$$

where $p$ and $q$ are parameters to control the relative importance of both functions. $h_{i j}$ is transformed into the weights of corresponding $\mu_{i j}$ to calculate the new membership function $\mu_{i j}^{\prime}{ }^{16}$. This approach has been successfully employed to improve FCM performance by utilizing image spatial information and reducing the negative effect from noise ${ }^{16,18,20,22,26}$. However, one major drawback of this approach is that the local noise filter is applied on each cluster of the membership function $\mu$ indiscriminately, hence when the clustering is not affected by the noise, the computational-intensive process of noise filtering becomes redundant, thus making the fuzzy clustering inefficient.

\subsection{Proposed Method}

\subsubsection{Rician Noise Detector}

It is known that noise in MR images follows Rician distribution ${ }^{25}$. Being referred to as the error between the underlying image intensities and the observed data, Rician noise has a local signal dependent mean, rather than Gaussian noise 25,28,29.

Wavelet-domain filtering can be employed to handle the spatial variations in both the signal and noise distribution. It is also demonstrated that the performance of noise removal method based on shrinkage of wavelet coefficients is asymptotic minimax-optimal and there is no excessive artifacts introduced during the signal reconstruction ${ }^{29,30}$. Wavelet coefficients can be used to obtain a detector of Rician noise level, or a threshold for discriminating noise and noise-free signals ${ }^{30}$.

The most commonly used approach to obtain threshold is through binary classification: a coefficient is dominated either by noisy or noise-free signal. If one threshold minimizes the mean square error $(M S E)$ of the wavelet coefficients of corrupted and uncorrupted image signals, the optimal signal to noise ratio $(S N R)$ becomes retrievable since the $M S E$ minimization is equivalent to $S N R$ maximization ${ }^{29}$.

In a wavelet decomposition of signals, a wavelet coefficient $w_{k, j}^{D}$ represents its bandpass content at resolution scale $2^{j} \quad(1 \leqslant j \leqslant J)$, spatial position $k$ and orientation $D$. Once a 2-dimensional wavelet transform is performed, the noise detector $d$ can be estimated by retrieving the median absolute deviation of the wavelet coefficients in the $H H$ subband at the finest resolution scale, divided by $0.6745^{30}$ as follows:

$$
d=\operatorname{Median}\left(\frac{\mid X-\text { med } \mid}{0.6745}\right)
$$

where $X$ is the wavelet coefficients at the finest resolution scale, and med stands for the median of $X$. The existence of noise can then be measured by comparing the noise detector $d$ with a threshold value. 


\subsubsection{Wavelet Denoising Filter}

Noisy signal can be modeled as $y=a+n$, where $a$ is the noise-free signal, $n$ is the noise ${ }^{31}$. With wavelet transformation, this noise model can be rewritten as follows:

$$
y_{o, s}(p)=a_{o, s}(p)+n_{o, s}(p)
$$

where $p$ is a wavelet coefficient at orientation $o$ and scale $s$, reused from $x$ in Equ. 5 .

The wavelet coefficients can be divided into two categories: wavelet coefficients that contain a signal of interest (hypothesis $H_{1}$ ) and those that do not (hypothesis $\left.H_{0}\right)^{32}$. The Minimum Mean Square Error (MMSE) of $a_{o, s}(p)$ can be retrieved as follows:

$$
\widehat{a}_{s}=E\left(a_{s} \mid y_{s}, H_{1}\right) P\left(H_{1} \mid y_{s}\right)+E\left(a_{s} \mid y_{s}, H_{0}\right) P\left(H_{0} \mid y_{s}\right)
$$

By assuming signal of interest is much higher than noise, we can respectively deduct $E\left(a_{s} \mid y_{s}, H_{1}\right) \approx y_{s}$ and $E\left(a_{s} \mid y_{s}, H_{0}\right) \approx 0$. Consequently, $M M S E$ can be rewritten as a general likelihood ratio as follows:

$$
\widehat{a}_{s}=P\left(H_{1} \mid y_{s}\right) y_{s}=\frac{1}{1+\eta\left(y_{s}\right)} y
$$

where $\eta\left(y_{s}\right)=\frac{p_{Y \mid H}\left(y_{s} \mid H_{0}\right)}{p_{Y \mid H}\left(y_{s} \mid H_{1}\right)}$ and $\xi=\frac{P\left(H_{0}\right)}{P\left(H_{1}\right)}$.

Image can be decomposed into 2 levels by multiresolution wavelet transform. We can then perform a non-linear shrinkage on the retrieved wavelet coefficients on each level and orientation.

Wavelet coefficient can be used to label signal of noise by calculating through the following equation:

$$
\widehat{a}_{k, s}^{D}= \begin{cases}0 & \text { if }\left|w_{k, s}^{D} \| \widehat{y}_{k, s+1}^{D}\right|<d \\ 1 & \text { if }\left|w_{k, s}^{D} \|\right| \widehat{y}_{k, s+1}^{D} \mid \geqslant d\end{cases}
$$

where $d$ is the reused noise detector in (5) and $w_{k, s}$ is the wavelet coefficients, $k$ is the index of wavelet coefficients.

The parameters of the generalized Laplacian prior can be estimated from the histogram of the wavelet coefficients. Then the probability density function can be estimated based on the histogram of $S_{0}=\left\{l: \widehat{a}_{l}=0\right\}$ and $S_{1}=\left\{l: \widehat{a}_{l}=1\right\}$. In order to reduce the effect of the errors in the tails, $\log$ function is used to fit the distribution ${ }^{27}$.

The likelihood $(p(m a g \mid 0)$ and $p(m a g \mid 1)$, where $m a g$ is the magnitude of the wavelet coefficient $w$ ) and the prior ratio $(p(e n g \mid 0)$ and $p(e n g \mid 1)$, where eng is the averaged energy of the neighboring coefficients) can thereby be computed. With the computed likelihood ratio and prior ratio, the probability that one coefficient contains signal of interest can be estimated as follows:

$$
\text { Probability }=\frac{\text { Likelihood } * r * \text { Prior_ratio }}{1+\text { Likelihood } * r * \text { Prior_ratio }}
$$

where $r$ can be retrieved by $\widehat{r}=\frac{\sum_{k=1}^{N} \widehat{a}_{k}}{N-\sum_{k=1}^{N} \widehat{a}_{k}} 27$.

We can then shrink the wavelet coefficients using Probability of significance. The denosing filter hence becomes effective since major signal of interest remains, while noise signal is reduced in the preceding process. The denosing process is finalized by performing the inverse wavelet transform to restore the image.

\subsubsection{Incorporating FCM with Noise Detector and Filter}

We can incorporate the Rician noise detector and filter into FCM by performing them on the membership function in each iteration of FCM clustering, instead of directly applying on the input image data 16. The algorithm employs the Rician noise detector to measure the existence of Rician noise reflected by the membership function, and selectively reduces noise with the denoising filter. The retrieved wavelet coefficients for Rician noise detector are reused for creating the denoising filter. The proposed FCM algorithm, which dynamically incorporates wavelet denosing filter, is referred to as dwFCM in the rest 
of the paper, and its outline is illustrated in Alg. 1.

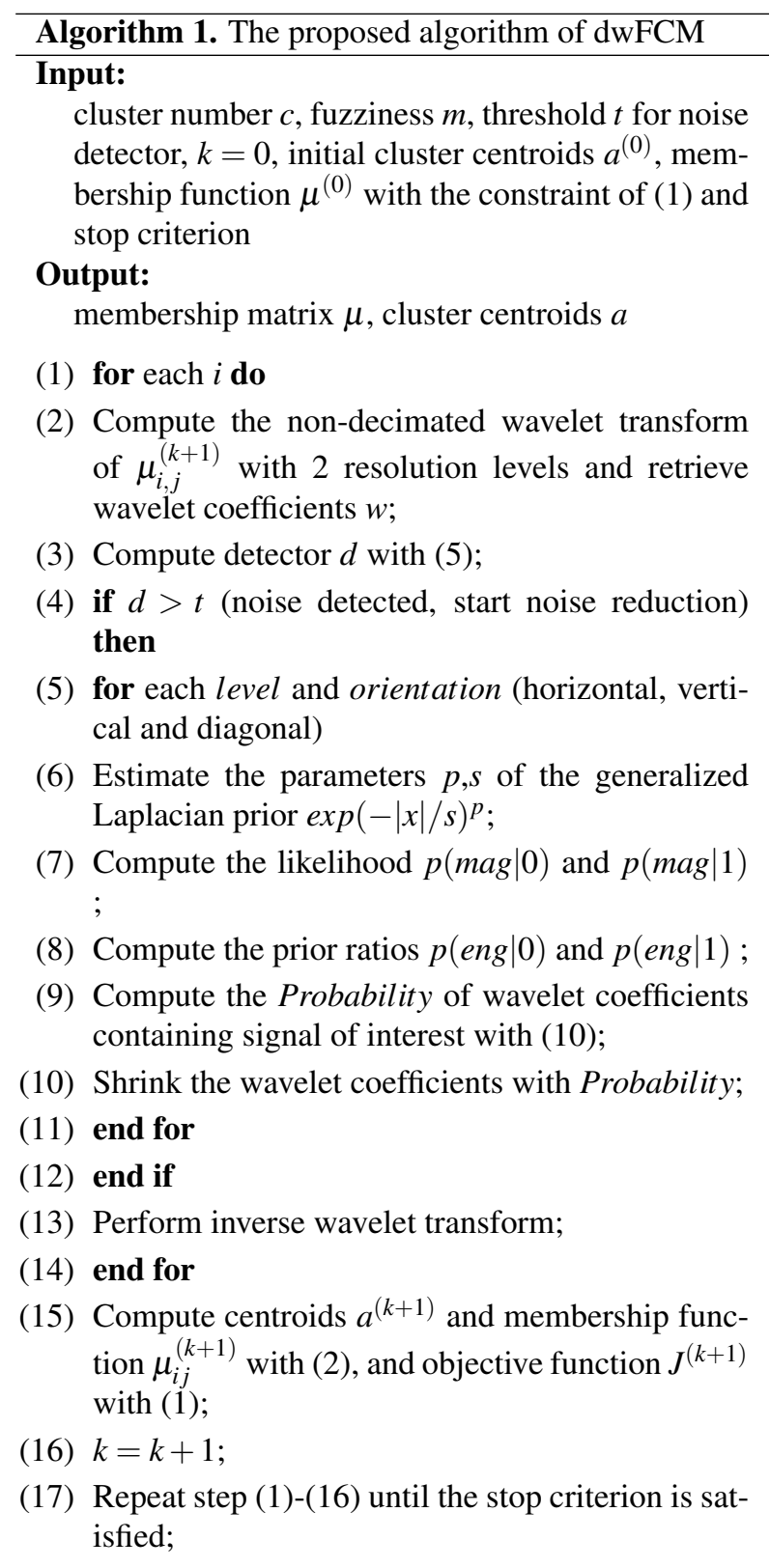

\section{Experimental Results}

In this section, we present comparative experiments with several existing FCM variants and the proposed algorithm. The algorithms are implemented by Mat- lab on a dual-CPU $2.5 \mathrm{GHz}$ Pentium PC with Windows 64 bit operating system. Throughout the experiments, for all the FCM variants, the maximum number of iteration is set to 100, parameters of feature weights to 1 , the parameter for controlling fuzziness $m$ in 1 to 2 , windows size of filters to $5 \times 5$, $\sigma$ for Gaussian filters to 0.94 , and cluster number to 6.

\subsection{Image Data and Visualized Clustering Results}

Both synthetic and brain MR images of the size of $256 \times 256$ are used in the experiments. A pair of synthetic images of smoothly distributed pixel intensity are shown in the first column in Fig. 1. Pixel intensity in the images linearly decreases from the top to bottom and left to right corner. The second and third columns in Fig. 1 illustrate the images corrupted by Rician noise of $S N R=15$ and $S N R=10$. Each pair (i.e., each column) of image data is employed as the multi-dimensional input data for the clustering algorithms.
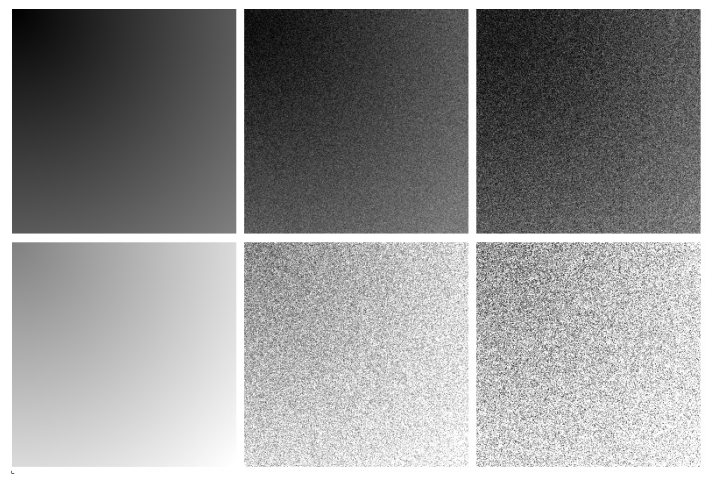

Fig. 1. Synthetic and the respective Rician noise-corrupted images: a pair of original images (first column), images respectively corrupted by noise of $S N R=15$ (second column), and by noise ratio of $S N R=10$ (third column)

We have conducted comparative experiments on the image data with standard FCM ${ }^{12}$, $\mathrm{sFCM}^{16}$, gFCM $^{18}$, bFCM $^{20}$, wFCM ${ }^{26}$ and the proposed dwFCM algorithm to evaluate the performance and efficiency of the proposed method from different perspectives.

Fig. 2 visualizes the synthetic image segmentation results by labeling each cluster with distinctive 
grayscale value. In the first row where no noise is added, all the clustering techniques create desirable segmentation results with smooth and clear edges, while in the second and third rows, where strong $\mathrm{Ri}$ cian noise of $S N R=15$ and 10 are respectively applied on the image, the added noise severely corrupts the segmentation results. It can be seen that, clustering results created by the use of FCM, sFCM, $\mathrm{gFCM}$, and $\mathrm{bFCM}$ as visualized respectively from the first to the fourth column contain multiple noisy and isolated spots, with blurry boundaries within clusters. It can be observed that in the fifth and sixth column where wFCM and dwFCM are applied, shaper boundaries of different clusters are created and multiple isolated spots are merged into blobs. In the third row, the stronger added noise further deteriorates segmentation results created by FCM, sFCM, gFCM and bFCM. In these cases, the boundaries within clusters are barely perceivable. While in the fifth and sixth columns, where wFCM and dwFCM are respectively used, isolated spots are further reduced, and boundaries between groups become more distinguishable.

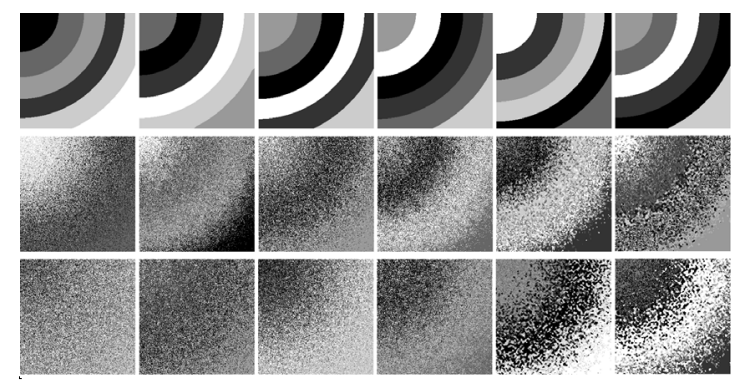

Fig. 2. Visualized clustering results on images from Fig. 2: original images(first row), images corrupted by noise of $S N R=15$ (second row) and images corrupted by noise of $S N R=10$ (third row), respectively by: FCM (first column), sFCM (second column), gFCM (third column), bFCM (fourth column), wFCM (fifth column) and dwFCM (sixth column)

The publicly available MICCAI BRATS Challenge (MBC) 2012 dataset ${ }^{\dagger}$ is also used for experiments. In this work, to take advantage of the infor- mation from multi-spectral MR images, we combine three images of T1-, T2-weighted and FLAIR images from each selected image slice set, thus creating 3-dimensional matrices as the input data set. We have selected 45 sets of image slices which contains pixels of all the types. As shown in Fig. 3 (b) and (d), images are corrupted by artificial Rician noise at various $S N R$ value to evaluate the performance and efficiency of the algorithm under different noise levels.

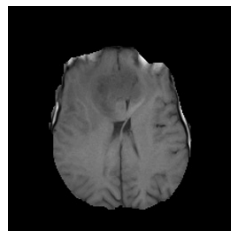

(a)

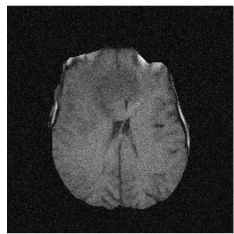

(d)

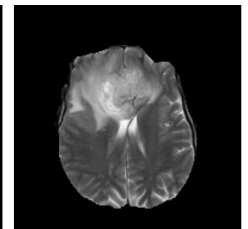

(b)

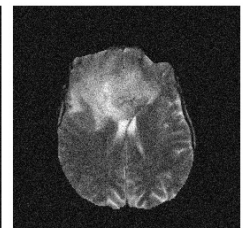

(e)

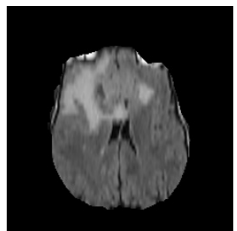

(c)

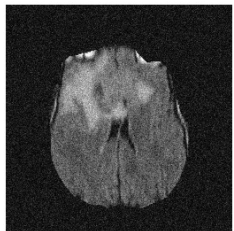

(f)
Fig. 3. MR images: (a) T1-weighted; (b) T2-weighted; (c) Flair; (d) Rician noise of $S N R=10$ on (a); (e) Rician noise of $S N R=10$ on (b); (f) Rician noise of $S N R=10$ on (c).

Fig. 4 visualizes the MR image segmentation results. The adopted clustering number of 6 is designed to accommodate cerebral organs of white matters (WM), gray matters (GM), cerebral-spinal fluid (CSF) along with background (BG), tumor and edema. It can be observed that when the image data is free of additive noise, most brain tissues are correctly separated by the standard FCM and all FCM variants. However when Rician noise of $S N R=10$ is added, segmentation results are interfered and becomes varied. Compared to other FCM variants, dwFCM not only merge the isolated points in the clusters of background and cerebral organs, but also create generally correct delineation of the WM and $\mathrm{GM}$, and contain less isolated pixels in these tissues. Further quantitative analysis results suggest the clus-

\footnotetext{
${ }^{\dagger}$ The brain-tumor image data used in this work were obtained from the MICCAI 2012 Challenge on Multimodal Brain Tumor Segmentation (http://www.imm.dtu.dk/projects/BRATS2012) organized by B. Menze, A. Jakab, S. Bauer, M. Reyes, M. Prastawa, and K. Van Leemput. This database contains fully anonymized images from the following institutions: ETH Zurich, University of Bern, University of Debrecen, and University of Utah.
} 
ters of intracranial tissues created by dwFCM contain the smallest amount of pixels affected by noise. By treating clusters of tissues from Fig. 4 (a) to (f) as masks, changed pixels in clusters caused by the additive Rician noise can be detected and counted. By performing the experiments on $328 \mathrm{MR}$ image slices, the average number of changed WM, GM and CSF pixels created by FCM, sFCM, gFCM, bFCM, wFCM and dwFCM are 3458.0, 2644.3, 2586.8, $1689.8,1328.3$ and 1279.1 , respectively. This clustering performance improvement can be attributed to the combination of FCM membership function and wavelet filter, which biases clustering solution toward homogeneous grouping.

\subsection{Performance in Terms of Computational Intensiveness}

A good clustering algorithm converges with minimum number of iteration and consumes less time in each iteration ${ }^{14}$. Fig. 5 illustrates the average number of iteration and computational time. Except the standard FCM, dwFCM algorithm spent the least amount of time; and in all the cases, average number of iteration with dwFCM is the smallest. It can be seen from Fig. 5 (b) that, compared with bFCM and gFCM, the efficiency gain of the dwFCM algorithm rises by more than 10 times when no noise or Rician noise of low intensity is added in the input image data. This significant enhancement can be attributed to the incorporated Rician noise detector, i.e., when Rician noise is not detected, the computational-intensive wavelet denoising process will be skipped. This effect can be further proved by the substantially increased time consumption by dwFCM when input images are corrupted by the strong additive noise of $S N R=10$. It should be noted that, even no additive noise added in the input images, performance of dwFCM could be different due to the fact that the Rician noise existing in the original image may be detected and filtered in certain iterations of dwFCM.
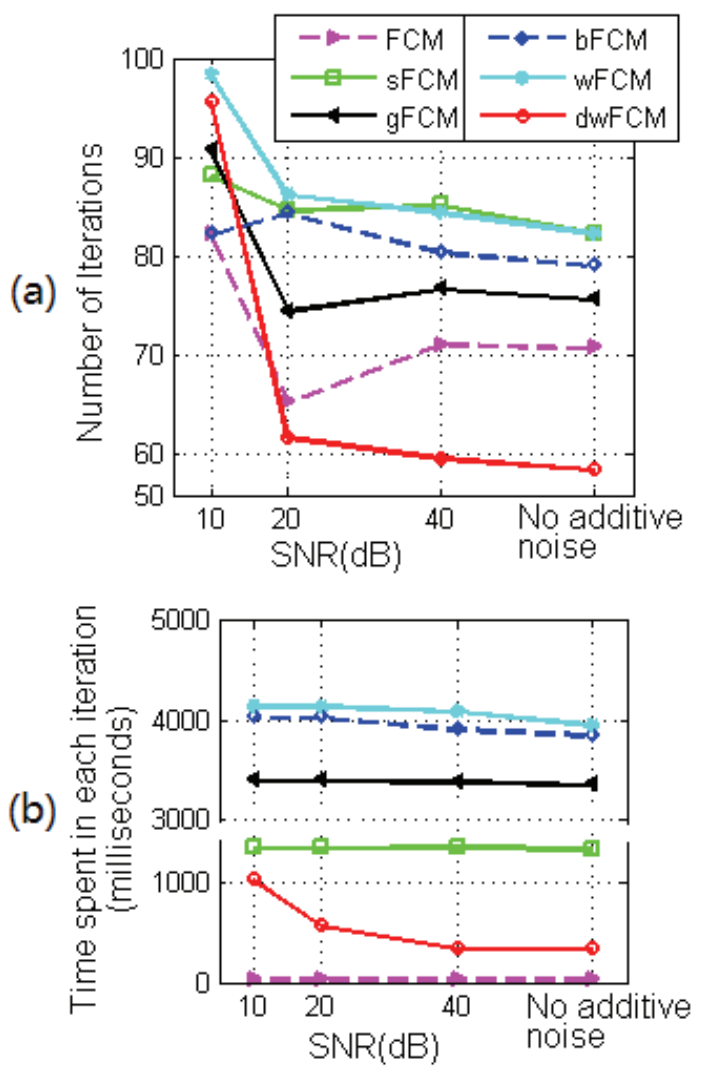

Fig. 5. Results of (a) iteration times; (b) time spent on each iteration by FCM variants with image data with additive $\mathrm{Ri}$ cian noise of different $S N R$.

\subsection{Performance in Terms of Validity Functions}

Validity functions based on fuzzy partition and feature structure are applied to evaluate the performance of the fuzzy clustering algorithms. Partition coefficient $V_{p c}{ }^{33}$ and partition entropy $V_{p e}{ }^{34}$, defined as (11), are the commonly used validity functions based on fuzzy partition. Less fuzziness means better performance, and therefore the best clustering is achieved when the value of $V_{p c}$ is maximal or $V_{p e}$ is minimal.

$$
V_{p c}=\frac{\sum_{j=1}^{N} \sum_{i=1}^{c} \mu_{i j}^{2}}{N} ; \quad V_{p e}=\frac{-\sum_{j=1}^{N} \sum_{i=1}^{c} \mu_{i j} \log \mu_{i j}}{N}
$$

Validity functions based on the feature structure 


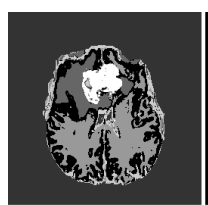

(a)

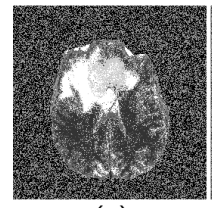

(g)

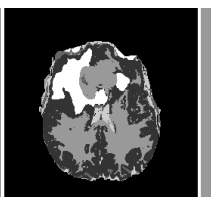

(b)

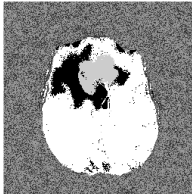

(h)

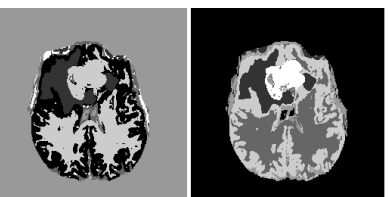

(c)

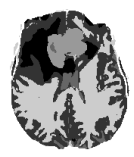

(e)

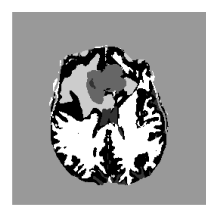

(f)

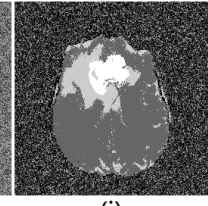

(i)

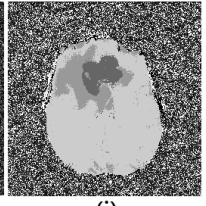

(j)

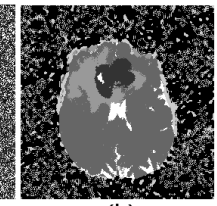

(k)

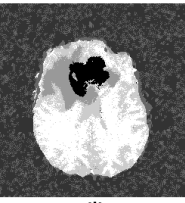

(I)

Fig. 4. Visualized clustering results on input image data with no additive noise by (a) FCM; (b) sFCM; (c) gFCM; (d) bFCM; (e) wFCM; (f) dwFCM, and added noise of $S N R=10$ by (g) FCM; (h) sFCM; (i) gFCM; (j) bFCM; (k) wFCM; (l) dwFCM.

35 makes a direct connection to the featuring property. $V_{x b}$ is the widely used one of this kind and defined as (12). Minimizing $V_{x b}$ is expected to lead to a good clustering ${ }^{35}$.

$$
V_{x b}=\frac{\sum_{j=1}^{N} \sum_{i=1}^{c} \mu_{i j}^{m}|| x_{j}-a_{i} \|^{2}}{N \times\left(\min _{1 \leqslant k, m \leqslant c, m \neq k}|| a_{k}-a_{m} \|^{2}\right)}
$$

Table 1 lists the average fuzzy validity function values of the multiple FCM variants using image data with Rician noise of different intensities. It can be seen that the stronger additive Rician noise, the lower $V_{p c}$ and the higher $V_{p e}$ and $V_{x b}$ will be created. This demonstrates the negative effect of the additive Rician noise on the fuzzy clustering performance. Meanwhile, compared to the standard FCM and all other FCM variants, wFCM and dwFCM create the highest $V_{p c}$ values and the lowest $V_{p e}$ and $V_{x b}$ values. This suggests that the incorporated wavelet filter in the membership function $\mu$ has more desired effect on removing Rician noises, and creates more homogeneous clustering for image data which obey Rician distribution. The advantage of the proposed $\mathrm{dwFCM}$ over our previous work of wFCM is also demonstrated in Table 1 that dwFCM creates better results than our previous work of wFCM.

\subsection{Performance in terms of brain tumor segmentation accuracy}

Although image clustering is not a preferred approach for brain tumor segmentation ${ }^{36}$, this research compares the results of FCM variants for brain tumor segmentation to study the applicability of the proposed dwFCM in more complex segmentation tasks. In this study, we have selected 142 sets of noticeable tumor-affected multi-spectral MR image slices, and respectively added noise of intensities of $S N R=40,20$ and 10 to the images.

Sensitivity (Sn) and Specificity $(S p){ }^{37}$ are applied for statistically measuring the tumor segmentation results. Using locations of ground truth (GT) tumor pixels as references (See Fig. 6), extracted pixels in the tumor cluster (See Fig. 7) can be labeled as correctly or wrongly segmented. And by treating correctly segmented tumor, wrongly segmented tumor, correctly segmented non-tumor and wrongly segmented non-tumor pixel number as true positive $\left(\right.$ true $\left.^{+}\right)$, false positive $\left(\right.$false $\left.^{+}\right)$, true negative $\left(\right.$ true $\left.^{-}\right)$and false negative ( fals $^{-}$) number respectively, $S n$ and $S p$ values can be obtained according to: $S n=\frac{\text { true }^{+}}{\text {true }^{+}+\text {false }^{-}}$and $S p=\frac{\text { true }^{-}}{\text {true }^{-}+\text {false }^{+}}$, respectively. An $S n$ of $100 \%$ means that the test recognizes all actual positives, i.e., all brain tumor pixels are segmented as tumor. And an $S p$ of $100 \%$ means that the test recognizes all actual negatives, i.e., all 
Table 1. Validity function values from various FCM variants and image data with Rician noise of different $S N R$

\begin{tabular}{|l|l|l|l|l|l|l|l|}
\hline \multicolumn{2}{|c|}{} & FCM & sFCM & gFCM & bFCM & wFCM & dwFCM \\
\hline \multirow{4}{*}{$V_{p c}$} & No additive noise & 0.853 & 0.924 & 0.925 & 0.926 & 0.930 & $\mathbf{0 . 9 3 2}$ \\
\cline { 2 - 8 } & Noise of $S N R=40$ & 0.847 & 0.924 & 0.925 & 0.926 & 0.930 & $\mathbf{0 . 9 3 1}$ \\
\cline { 2 - 8 } & Noise of $S N R=20$ & 0.691 & 0.823 & 0.826 & 0.839 & 0.869 & $\mathbf{0 . 8 0 0}$ \\
\cline { 2 - 8 } & Noise of $S N R=10$ & 0.503 & 0.646 & 0.657 & 0.662 & 0.670 & $\mathbf{0 . 6 7 3}$ \\
\hline \multirow{4}{*}{$V_{p e}$} & No additive noise & 0.296 & 0.144 & 0.142 & 0.140 & 0.133 & $\mathbf{0 . 1 2 9}$ \\
\cline { 2 - 8 } & Noise of $S N R=40$ & 0.305 & 0.144 & 0.141 & 0.141 & 0.132 & $\mathbf{0 . 1 3 0}$ \\
\cline { 2 - 8 } & Noise of $S N R=20$ & 0.557 & 0.302 & 0.297 & 0.280 & 0.233 & $\mathbf{0 . 2 2 1}$ \\
\cline { 2 - 8 } & Noise of $S N R=10$ & 0.923 & 0.594 & 0.587 & 0.572 & 0.566 & $\mathbf{0 . 5 6 2}$ \\
\hline \multirow{4}{*}{$V_{x b}$} & No additive noise & 0.010 & 0.013 & 0.013 & 0.013 & 0.004 & $\mathbf{0 . 0 0 2}$ \\
\cline { 2 - 8 } & Noise of $S N R=40$ & 0.010 & 0.013 & 0.013 & 0.013 & 0.004 & $\mathbf{0 . 0 0 2}$ \\
\cline { 2 - 8 } & Noise of $S N R=20$ & 0.013 & 0.016 & 0.016 & 0.016 & 0.007 & $\mathbf{0 . 0 0 4}$ \\
\cline { 2 - 8 } & Noise of $S N R=10$ & 0.034 & 0.033 & 0.037 & 0.037 & 0.034 & $\mathbf{0 . 0 3 3}$ \\
\hline
\end{tabular}

non-tumor pixels are segmented as non-tumor ${ }^{37}$.

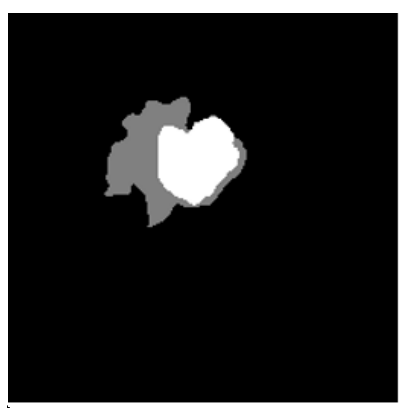

Fig. 6. With respect to image data of Fig 3, tumor (white area) and edema (gray area) as GT

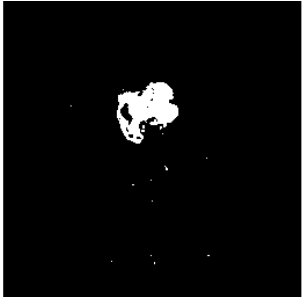

(a)

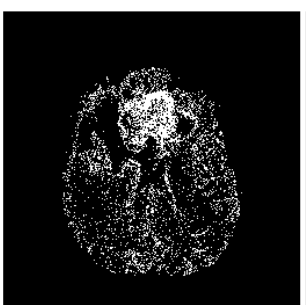

(c)

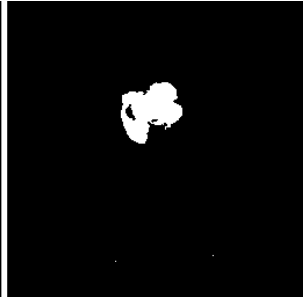

(b)

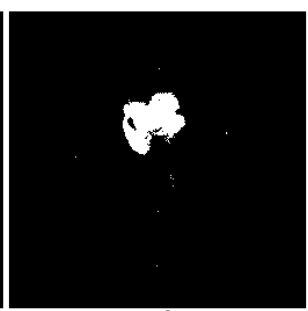

(d)
Fig. 7. With respect to image data of Fig 3, tumor segmentation on (a) no additive noise with FCM, (b) no additive noise with dwFCM, (c) added noise of $S N R=10$ with FCM, (d) added noise of $S N R=10$ with dwFCM.

It can be seen from the $S p$ and $S n$ values tabulated in Table 2 that all general FCM variants makes better tumor segmentation result than the standard FCM algorithm. The proposed dwFCM algorithm creates the best tumor segmentation accuracy when MR images are corrupted by noise of moderate in- 
Table 2. Tumor segmentation Specificity (Sp) and Sensitivity $(S n)$ produced by various FCM variants and image data with Rician noise of different $S N R$

\begin{tabular}{|c|l|l|l|l|l|l|l|}
\hline Intensity of added noise & & FCM & sFCM & gFCM & bFCM & wFCM & dwFCM \\
\hline \multirow{2}{*}{ None } & $S p$ & $94.85 \%$ & $96.49 \%$ & $95.99 \%$ & $96.40 \%$ & $96.37 \%$ & $\mathbf{9 6 . 9 3} \%$ \\
\cline { 2 - 7 } & $S n$ & $79.79 \%$ & $82.75 \%$ & $83.42 \%$ & $83.91 \%$ & $83.16 \%$ & $\mathbf{8 3 . 4 9} \%$ \\
\hline \multirow{2}{*}{$S N R=40$} & $S p$ & $93.84 \%$ & $94.41 \%$ & $94.18 \%$ & $94.44 \%$ & $94.70 \%$ & $\mathbf{9 4 . 8 9} \%$ \\
\cline { 2 - 7 } & $S n$ & $78.33 \%$ & $81.38 \%$ & $82.36 \%$ & $83.43 \%$ & $83.18 \%$ & $\mathbf{8 3 . 9 6} \%$ \\
\hline \multirow{2}{*}{$S N R=20$} & $S p$ & $85.29 \%$ & $90.72 \%$ & $91.19 \%$ & $91.34 \%$ & $91.57 \%$ & $\mathbf{9 1 . 6 8} \%$ \\
\cline { 2 - 7 } & $S n$ & $69.32 \%$ & $79.39 \%$ & $80.25 \%$ & $80.09 \%$ & $80.74 \%$ & $\mathbf{8 1 . 0 6} \%$ \\
\hline \multirow{2}{*}{$S N R=10$} & $S p$ & $71.05 \%$ & $79.88 \%$ & $79.13 \%$ & $\mathbf{8 0 . 0 7} \%$ & $79.26 \%$ & $79.40 \%$ \\
\cline { 2 - 7 } & $S n$ & $56.33 \%$ & $65.10 \%$ & $67.30 \%$ & $\mathbf{6 9 . 7 2} \%$ & $65.88 \%$ & $67.34 \%$ \\
\hline
\end{tabular}

tensities. When strong noise of $S N R=10$ is added in the MR images, both wFCM and dwFCM creates lower brain tumor segmentation than some FCM variants. This can be explained by the severely deteriorating effect from the strong noise, which substantially corrupted the input image data, thus making the tumor segmentation results unacceptable for real medical diagnosis or surgery preparation.

\section{Conclusion and Further Study}

The proposed dwFCM clustering algorithm for MR image segmentation is designed to deal with the $\mathrm{Ri}$ cian noise, which is common in MR images. By incorporating the wavelet-based Rician noise detector and filter in the membership function, the fuzzy clustering is capable of handling Rician distributed signal more efficiently and effectively. The computational intensiveness can be significantly decreased with the help of Rician noise detector which allows the algorithm bypass unnecessary noise filtering. The wavelet filter incorporated in the membership function can be used to remove the negative effect on the clustering performance from the Rician noise. One additional advantage of the proposed $\mathrm{dwFCM}$ is that it does not require any prior knowledge of the image.

In addition to the subjective observation and computation intensiveness evaluation, fuzzy validity functions and tumor segmentation accuracy measurements are applied for conducting discriminative and analytical experiments to evaluate the perfor- mance and robustness of the proposed method. The experimental results show that the proposed dwFCM algorithm has superior performance over existing filters-incorporated FCM variants, especially when moderate Rician noise is added. Future research will focus on improving the clustering performance of the dwFCM when Rician noise of high intensity is added in the image data.

Further study on improving the proposed approach aims on optimizing the wavelet denoising filter. The utilized wavelet denoising filter is proven robust and effective on MR images. However, in this study, the denoising filter is applied on the membership function matrix. We would design more effective wavelet denoising filters to adjust membership function based on the distribution of input image data.

\section{Acknowledgements}

The authors would like to acknowledge that this study was partly supported by a grant from the State Key Fundamental Research Program of China ("973" Program, No.2011CB711000).

\section{References}

1. J. Sled, A. Zijdenbos, and A. Evans, "A nonparametric method for automatic correction of intensity nonuniformity in mri data," IEEE Transactions on Medical Imaging, 17(1), 87-97 (1999).

2. L. P. Clarke, R. P. Velthuizen, M. A. Camacho, J. J. Heine, M. Vaidyanathan, L. O. Hall, R. Thatcher, and 
M. L. Silbiger, "Mri segmentation: methods and applications," Neuroanatomy, 11(3), 343-368 (1995).

3. M. F. Bear, B. W. Connors, and M. A. Paradiso, Neuroscience: Exploring the Brain, Lippincott Williams Wilkins, Philadelphia, PA, USA, 2006.

4. R. B. Gunderman, Essential Radiology: Clinical Presentation, Pathophysiology, Imaging, Thieme Medical Publishers, New York, NY, USA, 2006.

5. R. O. Duda, P. E. Hart, and D. G. Stork, Pattern Classification, Wiley-Interscience, New York, NY, USA, (2000).

6. J. H. Purba, and J. Lu, and G. Zhang, and W. Pedrycz, "A fuzzy reliability assessment of basic events of fault trees through qualitative data processing," Fuzzy Sets and Systems, 243, 50-69 (2014).

7. Zhang, G. and Ma, J. and Lu J, "Emergency management evaluation by a fuzzy multi-criteria group decision support system," Stochastic Environmental Research and Risk Assessment, 23(4), 517-527 (2009).

8. C. C. Meltzer, P. E. Kinahan, P. J. Greer, T. E. Nichols, C. Comtat, M. N. Cantwell, M. P. Lin, and J. C. Price, "Comparative evaluation of mr-based partial-volume correction schemes for pet," The Journal of Nuclear Medicine, 40(12), 2053-2065 (1999).

9. D. Gutierrez, M. Montandon, F. Assal, M. Allaoua, O. Ratib, K. Lvblad, and H. Zaidi, "Anatomically guided voxel-based partial volume effect correction in brain pet: impact of mri segmentation," Computerized Medical Imaging and Graphics, 36(8), 610-619 (2012).

10. J. Dunn, "A fuzzy relative of the isodata process and its use in detecting compact, well-separated clusters," Journal of Cybernetics, 3(3), 32-57 (1974).

11. J. Lu, J. Ma, G. Zhang, Y. Zhu, X. Zeng, and L. Koehl, "Theme-based comprehensive evaluation in new product development using fuzzy hierarchical criteria group decision-making method," IEEE Transactions on Industrial Electronics, 58(6), 2236-2246 (2011)

12. J. Bezek, R. Ehrlich, and W. Full, "Fcm: The fuzzy c-means clustering algorithm," Computers and Geosciences, 10(2-3), 191-203 (1984).

13. M. N. Ahmed, S. M. Yamany, N. Mohamed, A. A. Farag, and T. A. Moriarty, "Modified fuzzy c-means algorithm for bias field estimation and segmentation of MRI data," IEEE Transactions on Systems, Man, and Cybernetics, Part B: Cybernetics, 21, 193-199 (2002).

14. R. J. Almeida, and J. M. Sousa, "Comparison of fuzzy clustering algorithms for classification," Proc. of International Symposium Evolving Fuzzy Systems, 112117 (2006).

15. S. Asha, and R. S. Jeena, "Brain mr image segmentation using enriched fcm," International Journal of Scientific \& Engineering Research, 4(8), 14-17 (2013).

16. K. Chuang, H. Tzeng, S. Chen, J. Wu, and T.
Chen, "Fuzzy c-means clustering with spatial information for image segmentation," Computerized Medical Imaging and Graphic, 30(1), 9-15 (2006).

17. W. Cai, S. Chen, and D. Zhang, "Fast and robust fuzzy c-means clustering algorithms incorporating local information for image segmentation," Pattern Recognition, 40(3), 825-838 (2007).

18. K. Xiao, S. H. Ho, and A. E. Hassanien, "Automatic unsupervised segmentation methods for mri based on modified fuzzy c-means," Fundamenta Informaticae, 87(3), 465-481 (2008).

19. Z. Miao, C. Liu, and X. Lin, "FCM algorithm based on improved automatic segmentation of mri brain images," Computer Science for Environmental Engineering and EcoInformatics. Communications in Computer and Information Science, 159, 24-29 (2011).

20. Y. C. Liu, K. Xiao, A. L. Liang, and H. B. Guan, "Fuzzy c-means clustering with bilateral filtering for medical image segmentation," Proc. of Hybrid Artificial Intelligence Systems, 221-230 (2012).

21. N. Zhou, T. Yang, and S. Zhang, "An improved fcm medical image segmentation algorithm based on mmtd," Computational and Mathematical Methods in Medicine, vol. 2014, Article ID 690349, 8 pages, 2014. doi:10.1155/2014/690349.

22. I. Despotovic, E. Vansteenkiste, and W. Philips, "Spatially coherent fuzzy clustering for accurate and noiserobust image segmentation," IEEE Signal Processing Letters, 20(4), 295-298 (2013).

23. K. Xiao, J. L. Li, S. J. Xiao, and H. B. Guan, "Fuzzy clustering with multiresolution bilateral filtering for medical image segmentation," International Journal of Fuzzy System Applications, 3(4), 47-59 (2013).

24. R. Wagner, J. Smith, S. W. Sandrik, and H. Lopez, "Statistics of speckle in ultrasound b-scans," IEEE Transactions on Sonics and Ultrasonics, 30(3), 156163 (1983).

25. H. Gudbjartsson, and S. Patz, "The rician distribution of noisy mri data," Magnetic Resonance in Medicine, 34(6), 910-914 (1995).

26. S-L. Jui, C. Lin, H. B. Guan, A. Abraham, A. E. Hassanien, and K. Xiao, "Fuzzy c-means with wavelet filtration for MR image segmentation," Proc. of 6th World Congress on Nature and Biologically Inspired Computing, 12-16 (2014).

27. A. Pizurica, W. Philips, I. Lemahieu, and M. Acheroy, "A versatile wavelet domain noise filtration technique for medical imaging," IEEE Transactions on Medical Imaging, 22(3), 323-331 (2003).

28. A. Macovski, "Noise in mri," Magnetic Resonance in Medicine, 36(3), 494-497 (1996).

29. R. Nowak, "Wavelet-based rician noise removal for magnetic resonance imaging," IEEE Transactions on Medical Imaging, 8(10), 1408-1419 (1999).

30. D. L. Donoho, "De-noising by soft-thresholding," 
IEEE Transactions on Information Theory, 41(5), 613-627 (1995)

31. J. Sijbers, A. Dekker, P. Scheunders, and D. Dyck. , "Maximum-likelihood estimation of rician distribution parameters," IEEE Transactions on Medical Imaging, 17(3), 357-361 (1998).

32. H. Bhujle, and S. Chaudhur, "Laplacian based nonlocal means denoising of mr images with rician noise," Magnetic Resonance in Medicine, 31(9), 1599-1610 (2013).

33. J. Bezdek, "Cluster validity with fuzzy sets," Journal of Cybernetics, 3(1), 58-73 (1974).
34. J. Bezdek, "Mathematical models for systematic and taxonomy," Proc. of Eighth International Conference on Numerical Taxonomy, 143-166 (1975).

35. X. Xie, and G. Beni, "Validity measure for fuzzy clustering," IEEE Transactions on Pattern Analysis and Machine Intelligence, 3(1), 841-846 (1991).

36. J. Bezek, L. Hall, and L. Clarke, "Review of mr image segmentation using pattern recognition" Medical Physics, 20(4), 1033-1048 (1993).

37. D. G. Altman, and J. M. Bland, "Statistics notes: diagnostic tests 1 : sensitivity and specificity" $B M J, \mathbf{3 0 8}$, 1552 (1994). 